\title{
Características fisicoquímicas de la salchicha de cerdo del departamento de Tumbes, Perú
}

\author{
Characterization of a sausage produced in Tumbes, Peru \\ Daphne Ramos ${ }^{1}$, Viviana San Martín ${ }^{1}$, Mónica Rebatta ${ }^{1}$, Teresa Arbaiza ${ }^{1}$, Bettit Salvá2 Irma Caro $^{3}$ y Javier Mateo $^{3}$.
}

\section{RESUMEN}

Este trabajo pretende contribuir a la caracterización de la salchicha tradicional elaborada en Tumbes por pequeños productores. Se ha obtenido información de 12 productores sobre su procedimiento de elaboración y se ha analizado la composición ( $\mathrm{pH}, \mathrm{a}_{\mathrm{w}}$, componentes mayoritarios, ácidos láctico y acético, hidroxiprolina y estado oxidativo) y el color de 16 lotes de salchichas. Además, se ha estudiado en 6 lotes de salchichas la evolución de su composición y el momento de aparición de signos de alteración, durante 9 días de almacenamiento en las condiciones usuales de la zona (con periodos a temperatura ambiente y otros a refrigeración). La salchicha es un embutido fresco, entre húmedo y semiseco, con un contenido graso relativamente bajo y de baja acidificación. Hubo gran variabilidad entre muestras en los contenidos de componentes mayoritarios y $\mathrm{pH}$. La composición cambio poco durante el almacenamiento, pero se evidenció alteración sensorial a partir del tercer día.

Palabras claves: embutidos frescos; productos cárnicos; productos tradicionales; alimentos latinoamericanos

\section{SUMMARY}

The aim of this work was to contribute to the characterization of a sausage produced by small-scale producers in Tumbes (Peru). A total of 12 interviews were conducted with the correspondent producers in order to gather information about the process for producing this sausage, and sixteen sausage samples were analyzed for chemical composition $\left(\mathrm{pH}, \mathrm{a}_{\mathrm{w}}\right.$, proximal composition, lactic and acetic acids, hydroxyproline and oxidative state) and colour. Furthermore, during a 9-day storage, the changes in the sausage composition and the moment where the sausage was rejected by a sensorial panel (spoilage in odour, colour and/or texture) were assessed, using 6 branches of sausage the storage conditions were those usually followed in the area, which included storage periods at room temperature $\left(20-30{ }^{\circ} \mathrm{C}\right)$ and under refrigeration. The sausage from Tumbes can be defined as a wet or semi-dry, (relatively) lowfat and low-acid sausage. There was observed a great variability in the proximate composition and $\mathrm{pH}$ among the samples analyzed. Changes in the composition and color during its storage were scarce. However, some batches of sausage were rejected by the sensory panel after three days of storage.

Keywords: fresh sausage; meat products; traditional products; Latin American food

\section{INTRODUCCIÓN}

En el departamento de Tumbes, al norte del Perú se producen aproximadamente 400 toneladas métricas de carne de cerdo (Ministerio de Agricultura de Perú, 2009), el tipo de cerdo que predomina es el criollo de traspatio (Gómez, 2004). La mayor parte de los cerdos se destinan a su comercialización en vivo; sin embargo, algunos se sacrifican en la propia unidad productiva, obteniéndose carne fresca y/o preparados cárnicos frescos (principalmente salchicha y cecina) que se destinan al autoconsumo o la venta local a vecinos, en ferias locales o en pequeños establecimientos de servicio de comidas (The Cysticercosis Working Group in Peru, 1993). 
La salchicha de Tumbes es un embutido fresco popular en la zona, elaborado a partir de carne picada de cerdo por pequeños productores, siguiendo procedimientos y recetas familiares transmitidas de generación en generación (información obtenida de los propios productores), por lo que se puede considerar como un alimento tradicional (Guerrero et al., 2009). En su elaboración prácticamente no se usan aditivos ni ingredientes no cárnicos distintos a la sal, las especias y los condimentos, debido a que sólo utilizan los recursos disponibles en la zona, lo que está en contraste con la tendencia de elaborar embutidos frescos de bajo costo, que incluyen en su formulación proteínas no cárnicas y altos niveles de grasa, así como diversos aditivos (fosfatos, conservantes, saborizantes, colorantes, etc.), sustancias de relleno (almidones y gomas) entre otros (Feiner, 2006). Ante esta situación, se hace pertinente el establecimiento de criterios de calidad (niveles mínimos de proteína, contenidos máximos de grasa y humedad, etc.) que sirvan para regular el mercado y diferenciar los embutidos en categorías.

Aunque los embutidos frescos se deben almacenar y vender en condiciones de refrigeración (Feiner, 2006), la capacidad de los productores y vendedores locales de mantener la cadena de frío es limitada. Así pues, si la salchicha no se vende al día siguiente de su elaboración, se mantiene colgada a temperatura ambiente $\left(20-30{ }^{\circ} \mathrm{C}\right)$ por un tiempo variable que puede superar las 36 horas. El almacenamiento de embutidos frescos a temperatura ambiente acelera el crecimiento de microorganismos alterantes y posibilita el de ciertos patógenos, como Staphylococcus aureus enterotoxigénico o Salmonella spp. (Incze, 1992; Escartín et al., 1999; Ingham et al., 2009).

Debido a que no se han encontrado referencias bibliográficas sobre la salchicha elaborada en Tumbes y considerando el interés social y comercial de la caracterización de productos típicos y tradicionales en Latinoamérica, los objetivos de este estudio han sido:

- Presentar información sobre el proceso de elaboración de la salchicha de Tumbes

- Determinar los valores de diversos parámetros relevantes de su composición físico-química

- Estudiar los cambios en los parámetros físicoquímicos y sensoriales que tienen lugar durante el almacenamiento de la salchicha en las condiciones usualmente empleadas por los productores.

\section{MATERIALES Y MÉTODOS}

El presente trabajo consta de 3 etapas llevadas a cabo en el año 2007: la realización de encuestas para recabar información sobre el proceso tradicional de elaboración de la salchicha, su caracterización físicoquímica y el estudio de los cambios físico-químicos y sensoriales durante su almacenamiento. Las encuestas y las muestras de salchichas se tomaron de un total de 16 unidades productivas familiares, ubicadas en tres barrios de las afueras de la ciudad de Tumbes (barrios de Pueblo Joven, el Tablazo y Buenos Aires) y en dos villas de la provincia de Zarumilla (Uña de Gato y Realengal).

La recopilación de información sobre cómo se elabora la salchicha se llevó a cabo por medio de entrevistas personales de tipo estandarizado y abierto, estableciéndose las preguntas y su secuencia de antemano y permitiendo a los entrevistados dar una respuesta abierta (libre), interaccionando verbalmente el entrevistador con el entrevistado con el objeto de obtenerlamáximainformación. Seconsiguió entrevistar a los integrantes de 12 de las 16 unidades productivas anteriormente mencionadas. Se preguntó sobre: i) materias primas utilizadas (cantidades, regiones anatómicas empleadas, características de las tripas), ii) descripción del proceso de elaboración (tiempos, locales, utillaje) y iii) forma de almacenamiento y comercialización (características de los espacios, temperaturas, y tiempos de almacenamiento y canales de comercialización).

Por otra parte, se tomaron 16 muestras de salchicha (aproximadamente un $\mathrm{kg}$ de producto listo para $\mathrm{su}$ venta) de las respectivas unidades productivas. Cada muestra se cortó en 10 pedazos de unos $100 \mathrm{~g}$ cada uno y de ellos se eligieron tres al azar, que se colocaron en una bolsa con cierre hermético, introduciéndose en una caja térmica para su transporte al laboratorio. En el laboratorio se congelaron a $-40{ }^{\circ} \mathrm{C}$ hasta su posterior análisis. Los análisis llevados a cabo fueron los siguientes: humedad, proteína, cenizas, $\mathrm{NaCl}$, colágeno, actividad de agua $\left(\mathrm{a}_{\mathrm{w}}\right), \mathrm{pH}$, ácidos cítrico, láctico y acético, sustancias reactivas al ácido tiobarbitúrico (SRATB) y parámetros CIELab del color. Al momento de iniciar los análisis, las muestras se descongelaron a refrigeración, se les quitó la tripa y se molieron en un procesador de alimentos.

Para realizar el tercer experimento se pidió a 6 de las unidades productivas que preparen un lote de salchicha $(8-10 \mathrm{~kg})$ cada una y que lo mantuvieran hasta 9 días en sus locales, siguiendo sus prácticas habituales de almacenamiento hasta la venta. Así, durante el día las salchichas se mantuvieron colgadas 
en locales abiertos, al aire libre (patios), bajo la sombra (techos), a temperaturas entre $24-28{ }^{\circ} \mathrm{C}$ y humedades relativas entre el 80 y $88 \%$; al llegar la noche (7 de la noche, cuando acaban las ventas), las salchichas se colocaron en bandejas y se guardaron en un frigorífico doméstico a $5-10{ }^{\circ} \mathrm{C}$ hasta el día siguiente (6 de la mañana, cuando las ventas se reanudan), que se volvieron a colgar a temperatura ambiente y así sucesivamente hasta completar tres días. Del día 4 al día 9 de almacenamiento, las salchichas se mantuvieron en refrigeración permanentemente. Se realizó la toma de muestras en los días 1, 3, 6 y 9 de almacenamiento. El proceso de toma de muestras fue similar al descrito anteriormente y los análisis físicoquímicos realizados fueron: humedad, $\mathrm{NaCl}$ (solo el primer día), $\mathrm{a}_{\mathrm{w}}, \mathrm{pH}$, ácidos cítrico, láctico y acético, SRATB y parámetros CIELab del color. También se llevaron a cabo análisis sensoriales a partir de tres de los 10 pedazos en los que se dividió la muestra inicial, que se guardaron en bolsas y se congelaron a $-40{ }^{\circ} \mathrm{C}$ hasta el análisis.

La medición del $\mathrm{pH}$ se realizó sobre $5 \mathrm{~g}$ de muestra homogeneizada en $5 \mathrm{ml}$ de agua destilada con un $\mathrm{pH}-$ metro Crison modelo GLP 22 (Crison, Barcelona, España) y un electrodo de pH 52-02 de la misma marca. La medida de la $\mathrm{a}_{\mathrm{w}}$ se determinó en un equipo Aqualab CX-2 (Decagon Devices, Inc., Pullman, WA, EEUU). La humedad, grasa, proteína y cenizas fueron determinadas de acuerdo a los métodos recomendados por la AOAC (1999) - métodos oficiales 950.46, 991.36, 981.10 y 920.153 respectivamente. El colágeno fue determinado de acuerdo a la metodología descrita por la AOAC (1999) - Método oficial 990.26.

La extracción de $\mathrm{NaCl}$ se realizó según el método de Bruna et al (2003). Se partió de $10( \pm 0,01) \mathrm{g}$ de muestra a los que se adicionó $50 \mathrm{ml}$ de una solución de $\mathrm{H}_{2} \mathrm{SO}_{4} 4,5 \mathrm{mM}$, homogeneizando con un equipo Ultra-Turrax T18 (IKA, Werke GmbH y Co. KG, Staufen, Alemania) durante $1 \mathrm{~min}$ a $13500 \mathrm{rpm}$. El homogeneizado se llevó a un matraz y se enfrió durante $1 \mathrm{~h}$ a $5{ }^{\circ} \mathrm{C}$ y la mezcla se filtró primero a través de papel de filtro número 54 (Whatman International Ltd., Kent, Inglaterra) y después por una membrana de $0,45 \mathrm{~mm}$ de diámetro de poro número 184 (Sartorius Stedim Biotech GmbH, Alemania). La cuantificación se llevó a cabo por cromatografía líquida de alta resolución, en base al método descrito por Van Riel y Olieman (1986) con un instrumento de separación Alliance 2690 (Waters Corporation) equipado con una columna Aminex HPX-87H (Bio-Rad Laboratories, Inc., Hercules, CA, EEUU) con una precolumna
Cation $\mathrm{H}^{+}$Microguard (Bio-Rad Laboratories, Inc.) y detector de refractometría Waters 410 (Waters Corporation). El volumen de inyección fue de $15 \mu \mathrm{l}$, la fase móvil $\mathrm{H}_{2} \mathrm{SO}_{4} 5 \mathrm{mM}$, la velocidad de flujo 0,6 $\mathrm{ml}$ por min y la temperatura de la columna $60^{\circ} \mathrm{C}$. Para la cuantificación se realizó una curva de calibración usando soluciones patrón de $\mathrm{NaCl}$ (Sigma-Aldrich, St. Louis, MO, EEUU) que permitió determinar la concentración de la muestra en estudio.

El extracto anterior también se utilizó para la determinación de los ácidos láctico, acético y cítrico, en base al método descrito por Bruna et al. (2003). En la separación cromatográfica se empleó el instrumento y columna anteriormente mencionados, mientras que la detección se llevó a cabo a $210 \mathrm{~nm}$ con un detector de haz de diodos (Waters 996, Waters Corporation). El volumen de inyección fue de $30 \mu$ l, la fase móvil $\mathrm{H}_{2} \mathrm{SO}_{4}$ $3 \mathrm{mM}$, la velocidad de flujo fue de $0,5 \mathrm{ml}$ por min y la temperatura de la columna $65^{\circ} \mathrm{C}$. La identificación y cuantificación de los ácidos se llevó a cabo de forma similar a la mencionada para el $\mathrm{NaCl}$.

Las sustancias reactivas al ácido tiobarbitúrico (SRATB) de la salchicha se cuantificaron siguiendo el procedimiento descrito por Nam y Ahn (2003) con la salvedad que en el procedimiento utilizado la lectura en el espectrofotómetro fue llevada a cabo frente a un blanco con muestra, consistente en una solución preparada igual que las soluciones problema pero en la que se había excluido la adición de ácido 2-tiobarbitúrico.

La medida instrumental del color se realizó utilizando un colorímetro de reflectancia CM-500 (Minolta, Osaka, Japón) con iluminante D65 y $10^{\circ}$ de ángulo observador. Se tomaron unos $100 \mathrm{~g}$ de la masa de embutido, que fueron envueltos en un film plástico de polietileno, colocándose en la base de una placa de Petri de $90 \mathrm{~mm}$ de diámetro y $15 \mathrm{~mm}$ de altura. La masa se compactó con una presión de $2 \mathrm{~kg}$ durante 15 min y, después, se desprendió el film plástico de la superficie de la masa, realizándose las lecturas de color por triplicado. El equipo fue calibrado previamente usando una placa estándar blanca 677 T3 proporcionada por el fabricante.

Los análisis sensoriales se realizaron con un panel de 6 miembros previamente entrenados en análisis descriptivo del color, olor y textura de diversos productos cárnicos, entre ellos la salchicha. El entrenamiento específico fue enfocado en la alteración sensorial. Los evaluadores valoraron los 
atributos sensoriales típicos o normales asociados a un embutido fresco, recién elaborado y los atributos asociados a embutidos alterados, que en el caso de la salchicha fueron: color (empalidecido, oscurecimiento y manchas atípicas localizadas), olor (olor a notas aromáticas, a putrefacto, a rancio, a agrio $\mathrm{u}$ otros no definidos) y en cuanto a la textura (reblandecimiento, endurecimiento y limosidad superficial), apreciada por el tacto.

En cada sesión del análisis sensorial, se presentó a cada panelista unos $40 \mathrm{~g}$ de salchicha cruda (previamente descongelada a refrigeración). Las características a considerar fueron en este orden color, olor y textura, de la tripa y de la masa. Se pidió a los panelistas que valoraran en una escala de 2 valores $(0$ y 1) cada una de esas características en las muestras, otorgando la puntuación 1 cuando los atributos fueran los típicos o normales de un embutido fresco $\mathrm{y} / \mathrm{o}$ aceptables para su compra y consumo y 0 cuando fuesen inaceptables (merecedores de rechazo) para su compra y/o consumo. Además, en caso de rechazo se pidió a los evaluadores que describieran el motivo que lo justificara (descripción de la alteración). Las puntuaciones de los evaluadores se promediaron y se consideró que un atributo era inaceptable cuando su valor promedio era inferior a 0,5 .

\section{RESULTADOS Y DISCUSIÓN}

\section{Resultados de las encuestas realizadas a los productores de salchicha de Tumbes}

En base a las encuestas realizadas para recabar información sobre el proceso tradicional de elaboración de la salchicha, se pudo establecer el siguiente flujo de operaciones, que se detalla a continuación:

- La canal de cerdo se corta en cuartos (los delanteros son los más utilizados) y a estos se les que se quita la piel y se les deshuesa, obteniéndose piezas de carne sin separación entre músculo y grasa.

- La carne se pica manualmente con cuchillos o pequeñas picadoras con placas de 4 a $5 \mathrm{~mm}$ de diámetro y, después, se la mezcla con el resto de ingredientes mediante un amasado manual.

En el Cuadro 1 se detallan los ingredientes utilizados por 12 unidades productivas del departamento de Tumbes $(n=12)$ y la frecuencia de uso relativa de cada ingrediente (número de unidades productivas que utilizan dicho ingrediente en su formulación/total de unidades productivas), expresadas en tanto por uno. En dicho cuadro se puede apreciar que los ingredientes utilizados por la mayoría de las unidades productivas son carne, sal, pimienta, ajo, achiote, aceite, comino y limón.

- La masa se embute en intestino delgado, generalmente del cerdo sacrificado. Para lavar las tripas se elimina el contenido intestinal empujándolo con la mano hacia un extremo y luego los restos de ese contenido se arrastran con agua, en el cauce del río, en bateas o utilizando mangueras. A continuación el intestino se voltea, con ayuda de un palo de madera, y se elimina la mucosa y serosa frotando o raspando. Algunas personas frotan la tripa como si lavaran ropa, usando en algunos casos jabón o jugo de limón. Las tripas limpias se cortan en segmentos (0,5 a $1 \mathrm{~m}$ de longitud) y si no se usan en el momento se inflan y cuelgan para su secado al sol. En el llenado de las tripas, se amarra uno de los extremos con cuerda y por el otro se embute la masa cárnica a mano - a veces con ayuda de un embudo. Conforme se llena la tripa, manualmente se empuja (presiona) la masa hacia su fondo, desplazando y eliminando aire que pudiera quedar dentro y, a la vez, se pica la tripa con alfileres para contribuir a la eliminación del aire. Una vez llena la tripa se ata su extremo final.

-La mayor parte de las veces la salchicha se orea a la intemperie $\left(20-25^{\circ} \mathrm{C}\right)$, al abrigo del sol y la lluvia

Cuadro 1. Frecuencias relativas (tanto por uno) de uso de diversos ingredientes utilizados en la elaboración de la masa de la salchicha.

\begin{tabular}{|c|c|}
\hline Insumo & $\begin{array}{c}\text { Frecuencia } \\
\text { de uso } \\
(\mathrm{n}=12)\end{array}$ \\
\hline $\begin{array}{l}\text { Carne de cerdo (músculo, grasa } \\
\text { subcutánea e intermuscular) }\end{array}$ & 1,0 \\
\hline $\begin{array}{l}\text { Manteca fresca de cerdo (puede incluir } \\
\text { grasa omental) }\end{array}$ & 0,4 \\
\hline Sal & 1,0 \\
\hline Pimienta & 0,9 \\
\hline Ajo & 0,9 \\
\hline Achiote (Bixa orellana) & 0,8 \\
\hline Aceite vegetal & 0,7 \\
\hline Comino & 0,7 \\
\hline Limón (Citrus limonum) & 0,6 \\
\hline Glutamato monosodico & 0,5 \\
\hline Cebolla (Allium cepa) & 0,3 \\
\hline $\begin{array}{l}\text { Aliño (mezcla para salchicha preparada } \\
\text { y comercializada localmente) }\end{array}$ & 0,2 \\
\hline Vinagre & 0,2 \\
\hline Azúcar & 0,1 \\
\hline
\end{tabular}


hasta la mañana siguiente al día de su elaboración, que se expone colgada a la venta sin refrigeración (en ferias, mercados o pequeños establecimientos de servicio de comidas y bebidas). Si las salchichas no son vendidas ese día, en la noche se colocan en bandejas de plástico en un frigorífico o armario con hielo hasta la mañana siguiente, que se ponen a la venta de nuevo, repitiéndose el ciclo durante dos o tres días. Posteriormente las salchichas se mantienen en refrigeración de forma continua, destinándose a autoconsumo o venta vecinal.

\section{Caracterización fisicoquímica de la Salchicha de Tumbes}

En el Cuadro 2 se muestran algunos parámetros relacionados con la composición fisicoquímica de la salchicha de Tumbes, donde se puede observar

Cuadro 2. Parámetros relacionados con la composición fisicoquímica de la salchicha de Tumbes.

\begin{tabular}{|c|c|c|c|}
\hline Parámetro & $\begin{array}{l}\text { Media } \pm \text { DE } \\
\quad(\mathrm{n}=16)\end{array}$ & Mínimo & Máximo \\
\hline \multicolumn{4}{|l|}{$\begin{array}{l}\text { Componentes mayoritarios y diversos } \\
\text { parámetros calculados a partir de los } \\
\text { mismos (\%) }\end{array}$} \\
\hline Humedad & $51,90 \pm 7,87$ & 40,97 & 62,01 \\
\hline Grasa & $23,77 \pm 5,64$ & 15,92 & 32,91 \\
\hline Proteína & $16,74 \pm 3,22$ & 11,82 & 19,03 \\
\hline Cenizas & $3,18 \pm 0,89$ & 2,13 & 4,66 \\
\hline $\mathrm{NaCl}$ & $1,70 \pm 0,64$ & 0,73 & 2,97 \\
\hline $\begin{array}{l}\text { Humedad sobre extracto desengrasado } \\
(\% \mathrm{HED})^{\dagger}\end{array}$ & $68,5 \pm 4,8$ & 41,0 & 74,2 \\
\hline Tejido graso de la masa inicial ${ }^{\&}$ & $21,6 \pm 8,1$ & 11,3 & 36,0 \\
\hline \multicolumn{4}{|l|}{ Valor de $p H y a_{w}$} \\
\hline$a_{w}$ & $0,970 \pm 0,010$ & 0,951 & 0,987 \\
\hline $\mathrm{pH}$ & $5,56 \pm 0,36$ & 4,94 & 6,29 \\
\hline \multicolumn{4}{|l|}{ Ácidos de cadena corta (mg 100} \\
\hline Ácido cítrico & $60,4 \pm 89,0$ & $<1$ & 209,2 \\
\hline Ácido láctico & $458,3 \pm 257,1$ & 204,6 & 901,1 \\
\hline Ácido acético & $66,5 \pm 55,6$ & $<2$ & 165,6 \\
\hline \multicolumn{4}{|l|}{ Otros parámetros de composición } \\
\hline Hidroxiprolina (\%) & $0,38 \pm 0,17$ & 0,27 & 0,62 \\
\hline $\begin{array}{l}\text { Sustancias reactivas al ácido } \\
\text { tiobarbitúrico }\left(\mathrm{mg} \mathrm{kg}^{-1}\right)\end{array}$ & $2,73 \pm 2,60$ & 0,40 & 8,40 \\
\hline \multicolumn{4}{|l|}{$\underline{\text { Color }}$} \\
\hline$L^{*}$ & $44,84 \pm 5,44$ & 37,67 & 52,90 \\
\hline$a^{*}$ & $23,10 \pm 7,62$ & 14,95 & 41,02 \\
\hline$b^{*}$ & $36,63 \pm 9,35$ & 22,23 & 49,08 \\
\hline
\end{tabular}

- El \%HED es un parámetro relacionado con el grado de secado, considerándose un valor de 77,0\% para un embutido de cerdo recién elaborado (Prieto y Carballo, 1997).

- Valor estimado a partir del porcentaje de grasa (química) del embutido multiplicado por su \%HED y por 100 y dividido entre 77 (Prieto y Carballo, 1997).

- Valor obtenido multiplicando la proteína del embutido por 4,8 (100 entre 20,8, que es el porcentaje de proteína de la carne magra de cerdo; Feiner, 2006). 
Cuadro 3. Cambios en la composición de la salchicha de Tumbes durante el periodo de almacenamiento.

\begin{tabular}{|c|c|c|c|c|}
\hline & $\begin{array}{l}\text { Día 0 } \\
(\mathrm{n}=6)\end{array}$ & $\begin{array}{l}\text { Día 3 } \\
(\mathrm{n}=6)\end{array}$ & $\begin{array}{l}\text { Día } 6 \\
(\mathrm{n}=6)\end{array}$ & $\begin{array}{l}\text { Día } 9 \\
(\mathrm{n}=6)\end{array}$ \\
\hline \multicolumn{5}{|c|}{ Componentes mayoritarios (\%) } \\
\hline Humedad (\%) & $58,73 \pm 11,08^{\mathrm{a}}$ & $52,16 \pm 7,44^{\mathrm{a}}$ & $52,26 \pm 6,92^{\mathrm{a}}$ & $53,71 \pm 6,56^{\mathrm{a}}$ \\
\hline $\mathrm{NaCl}(\%)$ & $1,17 \pm 0,40$ & - & - & - \\
\hline \multicolumn{5}{|c|}{ Valor de $p H y a_{w}$} \\
\hline$a_{w}$ & $0,984 \pm 0,006^{\mathrm{a}}$ & $0,974 \pm 0,005^{\mathrm{b}}$ & $0,973 \pm 0,004^{b}$ & $0,973 \pm 0,007^{\mathrm{b}}$ \\
\hline $\mathrm{pH}$ & $5,70 \pm 0,72^{\mathrm{a}}$ & $5,41 \pm 0,35^{\mathrm{a}}$ & $5,44 \pm 0,49^{\mathrm{a}}$ & $5,58 \pm 0,55^{\mathrm{a}}$ \\
\hline \multicolumn{5}{|c|}{ Ácidos de cadena corta $\left(m g 100 g^{-1}\right)$} \\
\hline Ácido cítrico & $145,0 \pm 106,3^{\mathrm{a}}$ & $44,9 \pm 62,8^{\mathrm{a}}$ & $69,2 \pm 77,9^{\mathrm{a}}$ & $70,3 \pm 80,0$ \\
\hline Ácido láctico & $412,3 \pm 135,9^{\mathrm{b}}$ & $606,8 \pm 318,2^{\mathrm{ab}}$ & $844,8 \pm 153,6^{\mathrm{a}}$ & $879,3 \pm 190,6^{\mathrm{a}}$ \\
\hline Ácido acético & $16,8 \pm 41,1^{\mathrm{b}}$ & $86,6 \pm 48,5^{\mathrm{a}}$ & $123,5 \pm 52,8^{\mathrm{a}}$ & $87,3 \pm 32,7^{\mathrm{a}}$ \\
\hline \multicolumn{5}{|c|}{ Sustancias reactivas al ácido tiobarbitúrico (SRATB) } \\
\hline SRATB & $2,21 \pm 2,19^{\mathrm{a}}$ & $2,24 \pm 2,34^{\mathrm{a}}$ & $1,83 \pm 2,38^{\mathrm{a}}$ & $1,76 \pm 2,96^{\mathrm{a}}$ \\
\hline \multicolumn{5}{|l|}{ Color } \\
\hline$L^{*}$ & $48,32 \pm 5,17^{\mathrm{a}}$ & $42,83 \pm 5,86^{\mathrm{a}}$ & $45,14 \pm 5,05^{\mathrm{a}}$ & $43,18 \pm 7,07^{\mathrm{a}}$ \\
\hline$a^{*}$ & $21,67 \pm 6,42^{\mathrm{a}}$ & $23,23 \pm 5,68^{a}$ & $23,50 \pm 5,15^{\mathrm{a}}$ & $23,64 \pm 6,43^{a}$ \\
\hline$b^{*}$ & $44,30 \pm 7,42^{\mathrm{a}}$ & $41,24 \pm 4,39^{\mathrm{a}}$ & $43,47 \pm 4,77^{\mathrm{a}}$ & $39,58 \pm 3,98^{\mathrm{a}}$ \\
\hline
\end{tabular}

Cuadro 4. Frecuencia acumulada de aparición de diversas alteraciones sensoriales en la salchicha de Tumbes durante su almacenamiento.

\begin{tabular}{lcccc}
\hline & $\begin{array}{l}\text { Día 0 } \\
(\mathrm{n}=6)\end{array}$ & $\begin{array}{c}\text { Día 3 } \\
(\mathrm{n}=6)\end{array}$ & $\begin{array}{c}\text { Día } 6 \\
(\mathrm{n}=6)\end{array}$ & $\begin{array}{c}\text { Día } 9 \\
(\mathrm{n}=6)\end{array}$ \\
\hline $\begin{array}{l}\text { Alteración del color } \\
\quad \text { Tripa }\end{array}$ & 0 & $2 / 6$ & $3 / 6$ & $3 / 6$ \\
$\quad \begin{array}{l}\text { Masa } \\
\text { Alteración del olor } \\
\quad \text { Masa y tripa }\end{array}$ & 0 & $0 / 6$ & $2 / 6$ & $2 / 6$ \\
$\begin{array}{l}\text { Alteración de la textura al tacto manual } \\
\text { Tripa }\end{array}$ & 0 & $2 / 6$ & $3 / 6$ & $4 / 6$ \\
$\quad$ & 0 & $1 / 6$ & $1 / 6$ & $6 / 6$ \\
Masa & 0 & 0 & $1 / 6$ & $5 / 6$ \\
\hline
\end{tabular}

una variabilidad considerable que puede atribuirse a diferencias en el grado de secado y en las cantidades de carne magra, grasa y sal de la masa inicial. Por su contenido en humedad (entre 40 y $50 \%$ ) y cociente humedad/proteína (H/P; entre 2,3 y 3,4), la mitad de las salchichas analizadas se clasifican como embutidos semisecos, con pérdidas de peso entre el 10 y $20 \%$, y la otra mitad serían no desecados (Adams, 1986).

Para el cálculo de las medias de las concentraciones de los ácidos cítrico y acético, cuando la medida estuvo por debajo del límite de detección su valor se consideró como cero.

Los niveles humedad, grasa y proteína de la salchicha de Tumbes fueron similares a los descritos para algunos embutidos frescos latinoamericanos (Reyes et al. 2009; Austria et al., 2006). No obstante, el contenido en grasa fue inferior al rango de 27 $-34 \%$ reportado en otros embutidos frescos de Perú (INDECOPI, 1999; Bejarano et al., 1993; Reyes et al., 2009), entre los que se incluye la popular salchicha de 
Huacho. Este relativamente escaso contenido de grasa probablemente obedezca al uso de carne de cerdos de traspatio, con baja cantidad de grasa corporal (RamosDelgado, 2008). Por otra parte, el contenido medio de sal en la salchicha $(1,7 \%)$ concuerda con el rango usual de sal agregada $(1,4-1,6 \%)$ a la masa de los embutidos frescos (Feiner, 2006).

Casi todas las salchichas tuvieron un $\mathrm{pH}$ mayor a 5,3 (Cuadro 2), por lo que se pueden clasificar como embutidos de baja acidificación (Incze, 1992). La variabilidad en el $\mathrm{pH}$ se puede atribuir a diferencias en el $\mathrm{pH}$ de la carne, a la adición o no de limón o vinagre a la masa o al grado de fermentación láctica (ver las desviaciones estándar para los ácidos cítrico, acético y láctico en el Cuadro 2). Respecto al último factor, no obstante, como el contenido promedio de ácido láctico de la salchicha de Tumbes fue similar al de la carne de cerdo (0,6\%; Simek et al., 2003), cabe pensar que la actividad fermentativa fue reducida.

El contenido de hidroxiprolina en la salchicha fue de $0,38 \%(0,75 \%$ si se expresa sobre materia seca). Esta cantidad permite clasificar a este embutido en la penúltima categoría de calidad de las cuatro establecidas en la legislación española para embutidos crudo-curados (Presidencia del Gobierno de España, 1980). No obstante, se ha de tener en cuenta que la salchicha de Tumbes, a diferencia de los embutidos crudo curados, se consume cocinada, por lo que la cantidad de colágeno probablemente tiene menos repercusiones negativas sobre su dureza, que las que pueda tener en los crudo-curados. Por otra parte, el nivel promedio de las sustancias reactivas al ácido tiobarbitúrico (SRATB) (Cuadro 2) estuvo en el límite del umbral para la detección sensorial de rancidez en carne fresca (3 $\mathrm{mg} \mathrm{kg}^{-1}$; Fernández et al., 1997).

Los valores de $L^{*}$ y $a^{*}$ determinados en la salchicha de Tumbes (Cuadro 2) estuvieron dentro de los rangos encontrados en la bibliografía para diversos chorizos frescos españoles (también de color rojo): $L^{*}, 30$ 50; $a^{*}$ y 16-26 (Ansorena et al., 1997; Gómez et al., 2001). Respecto al parámetro $b^{*}$, su valor fue superior al rango correspondiente (14-29). Esta discrepancia se puede atribuir a que el color de la salchicha de Tumbes no solo depende la carne y el pimiento, sino también del achiote.

\section{Cambios durante el almacenamiento de la Salchicha de Tumbes.}

Durante el almacenamiento de las salchichas solo se observaron cambios destacables en la humedad, $\mathrm{a}_{\mathrm{w}}$, ácidos láctico y acético y luminosidad (Cuadro 3).
El contenido de humedad y la $\mathrm{a}_{\mathrm{w}}$ disminuyeron durante los tres primeros días para luego estabilizarse hasta el final del periodo estudiado (9 días). El descenso inicial se atribuye al secado de la salchicha cuando está permanecía colgada al aire y la estabilización posterior al hecho de que en todo ese tiempo la salchicha se mantuvo apilada en bandejas en el refrigerador. El incremento de ácido láctico observado durante el almacenamiento de las salchichas es indicativo de que hubo fermentación de los azúcares presentes por las BAL. No obstante, esa fermentación fue poco acusada probablemente por una escasa cantidad de azúcares. Como es de esperar en los procesos fermentativos de los embutidos, el incremento de ácido láctico se ve acompañado de un aumento en el ácido acético (Lücke, 1994; Demeyer et al., 2000). Finalmente, la luminosidad $\left(L^{*}\right)$ de la salchicha mostró un comportamiento similar al de la humedad, por lo que se podría establecer una relación entre ambos parámetros.

Los cambios en las características sensoriales de la salchicha fueron evidentes (Cuadro 4). El panel de evaluadores rechazó a los tres días de almacenamiento dos de los seis lotes estudiados y en el día 9 todos los lotes. Los motivos de rechazo del color fueron la pérdida de color típico en la tripa (empalidecido y opacidad) y la pérdida parcial del color rojo de la masa, que adquirió una tonalidad más marrón, probablemente por oxidación de los pigmentos. Por otra parte, los del olor fueron el olor a putrefacción y otros olores desagradables no definidos. Finalmente, los de textura fueron la limosidad en superficie y masa reblandecida. La elevada $\mathrm{a}_{\mathrm{w}}$ de la salchicha, $\mathrm{su} \mathrm{pH}$ similar al de la carne junto con las horas que ésta se mantiene a temperatura ambiente en los primeros días de almacenamiento provocan un rápido crecimiento microbiano alterante (Incze, 1992; Nychas et al., 2008), hasta llegar a ocasionar las alteraciones mencionadas, y además permiten el crecimiento de microorganismos patógenos (Ingham et al., 2009).

\section{CONCLUSIONES}

La salchicha elaborada en Tumbes presenta una gran variabilidad en su composición química.

La salchicha, almacenada según las condiciones comúnmente seguidas en la zona, tiene una vida útil muy corta (3-6 días).

Se sugiere investigar el efecto de la adición de azúcares (o ingredientes ricos en azúcares) a la masa del embutido sobre su vida útil y calidad sensorial, debido a que una mayor cantidad de azúcares permitiría incrementar la producción de ácido láctico en dicho producto y mejorar su conservación. 
Los resultados de este estudio son una referencia para llevar a cabo actividades de capacitación, elaboración de estándares de calidad y búsqueda de alternativas que mejoren las condiciones de procesado y almacenamiento de la salchicha tradicional de Tumbes.

\section{Agradecimientos}

Agradecemos la financiación de la Agencia Española de Cooperación Internacional para el Desarrollo (proyectos A/5909/06 y A/011790/07) y la Fundación Bill y Melinda Gates (Proyecto: A demonstration project to eliminate cysticercosis in Peru and thus develop a model by which the disease may be eradicated in other parts of the world CC80147) y al Cysticercosis Working Group in Perú por su apoyo.

\section{Correspondencia:}

\section{Daphne Ramos Delgado}

dramosd@unmsm.edu.pe

\section{REFERENCIAS BIBLIOGRÁFICAS}

1. Adams MR. 1986. Fermented flesh foods. En: Adams MR.(Ed.), Progress in industrial Microbiology. Amsterdam: Elsevier. p. 159-198.

2. Ansorena D, Peña MP, Astiasarán I; Bello, J. 1997. Colour evaluation of chorizo de Pamplona, a Spanish dry fermented sausage: comparison between the CIE $L^{*} a^{*} b^{*}$ and the Hunter Lab Systems with illuminants $\mathrm{D}_{65}$ and C. Meat Science 46: 313-318.

3. American Official Of Analytical Chemists. AOAC. 1999. Official method 981.10 crude protein in meat. Block digestion method; official method 991.36 fat (crude) in meat and meat products; Official method 950.46 moisture in meat; official method 920.153 ash in meat. Block digestion method. Official method 990.26 hydroxyproline in meat and meat products. Meat and meat products. In CUNNIFF, P. (Ed.), Official methods of analysis of the AOAC international. $16^{\text {th }}$ ed. Washington,

4. Austria V, Soto S, Caro I, Fonseca B, Güemes N, Mateo J. 2006. Composition and physiochemical characteristics of "chorizo" from the hidalgense huasteca region in Mexico. In: 52nd International Congress of Meat Science and Technology, 8, 2006, Dublin, Ireland, Proceedings. The Netherlands: Wageningen Academic. p.423-424.

5. Bejarano E, Bravo M, Huamán M, Huapaya $C$, Roca A, Rojas E. 1993. Tabla de composición de alimentos industrializados. Ministerio de Salud, Instituto Nacional de Salud, Centro Nacional de Alimentación y Nutrición. Lima. Perú.

6. Bruna JM, Hierro EM, de la Hoz L, Mottram DS, Fernández M, Ordoñez JA 2003. Changes in selected biochemical and sensory parameters as affected by the superficial inoculation of Penicillium camemberti on dry fermented sausages. International Journal of Food Microbiology 85: 111-125.

7. Demeyer M, Raemakers A, Rizzo A, Holck A, Smedt B, Brink B, Hagen B, Montel C, Zanardi E, Murbrekk E, Leroy F, Vandendriessche F, Lorentsen K, Venema K, Sunensen L, Stahnke L, Vuyst L, Talon R, Chizzolini R, Eerola S. 2000. Control of bioflavour and safety in fermented sausages: first results of a European proyect. Food Research International 33: 171-180.

8. Escartin EF, Castillo A, Hinojosa-Puga A, SaldañaLozano J. 1999. Prevalence of Salmonella in chorizo and its survival under different storage temperatures. Food Microbiology 16: 479-486.

9. Feiner G. (2006). Fresh sausages. En: Feiner G. (Ed.), Meat Products Handbook Practical Science and Technology. Cambridge, England: Woodhead Publising Limited, p.297-309.

10. Fernández J, Pérez-Álvarez JA, Fernández-López JA. (1997). Thiobarbituric acid test for monitoring lipid oxidation in meat. Food Chemistry 59: 345-353.

11. Gómez M. 2004. Situación actual y descripción del porcino criollo peruano. En: Delgado-Bermejo JV. (Ed.), Biodiversidad Porcina Iberoamérica: Caracterización y Uso Sustentable. Córdoba, España: Universidad de Córdoba. p.191-200.

12. Gómez R, Picazo MI, Alvarruiz A, Pérez JI, Valera D, Pardo JE. 2001. Influencia del tipo de pimentón en la pérdida de color del chorizo fresco. Alimentaria 323: 67-73.

13. Guerrero L, Guardia MD, Xicola J, Verbeke W, Vanhonacker F, Zakowska-Biemans S, Sajdakowska M, Sulmont-Rosse C, Issanchou S, Contel M, Scalvedi ML, Granli BS, Hersleth M. 2009. Consumer-driven definition of traditional food products and innovation in traditional foods. A qualitative cross-cultural study. Appetite 52: 345-354.

14. Incze K. 1992. Raw fermented and dried meat products. Fleischwirtschaft 72: 58-62.

15. INDECOPI. Instituto Nacional de Defensa del Consumidor y de la Protección de la Propiedad Intelectual. 1999. Norma Técnica Peruana NTP 201.012-1999: Carne y productos cárnicos. Embutidos Crudos. Definiciones, clasificación y requisitos. Perú: INDECOPI, $11 \mathrm{p}$.

16. Ingham SC, Ingham BH, Borneman D, Jaussaud E, Schoeller EL, Hoftiezer N, Schwartzburg L, Burnham GM, Norback JP. 2009. Predicting pathogen growth during short-term temperature abuse of raw sausage. Journal of Food Protection 72: 75-84.

17. LÜCKE FK. 1994. Fermented meat products. Food 
Research International 27: 299-307.

18. NAM KC, AHN DU. 2003. Combination of aerobic and vacuum packaging to control lipid oxidation and off-odor volatiles of irradiated raw turkey breast. Meat Science 63: 389-395.

19. NYCHAS, G.J.E.; SKANDAMIS, P.N.; TASSOU, C.C.; KOUTSOUMANIS, K.P. 2008. Meat spoilage during distribution. Meat Science (78), 77-89.

20. Ministerio de Agricultura de Perú. 2009. Estadística agraria mensual, noviembre 2009. Oficina de Estudios Económicos y Estadísticos. Disponible en: http:// www.minag.gob.pe/boletines/estadistica-agrariamensual.html

21. Presidencia del Gobierno de España. 1980. Norma de calidad para los productos cárnicos embutidos crudos-curados en el mercado interior. Disponible en: http://www.boe.es/boe/dias/1980/03/21/pdfs/ A06280-06284.pdf

22. Prieto B, Carballo J. 1997. El control analítico de la calidad en los productos cárnicos crudos-curados. Ciencia y Tecnología Alimentaria 1: 112-120.

23. Ramos-Delgado D. (2008). Caracterización de la canal y la carne del cerdo criollo y de los productos cárnicos en el Departamento de Tumbes-Perú. Tesis de Ph.D. Universidad de León, España. 345p.
25. Reyes M, Gómez-Sánchez I, Espinoza C, Bravo F, Ganoza L. 2009. Tablas peruanas de composición de alimentos. Disponible en: http:/www.ins.gob. pe/insvirtual/images/otrpubs/pdf/Tabla $\% 20 \mathrm{de} \% 20$ Alimentos.pdf

26. Simek J, Vorlova L, Malota L, Steinhauserova I, Steinhauser L. 2003. Postmortem changes of $\mathrm{pH}$ value and lactic acid content in the muscles of pigs and bulls. Czech Journal of Animal Science 48: 295299.

27. The Cysticercosis Working Group in Peru. 1993. The marketing of cysticercotic pigs in the sierra of Peru. Bulletin of the World Health Organization 71: 223-228.

28. van Riel JA, Olieman C. 1986. High-performance liquid chromatography of sugars on a mixed captionexchange resin column. Journal of Chromatography A $362: 235-242$.

Recibido 17/11/2014

Aceptado 09/02/2015 